Proceedings of the

IEEE Intelligent Vehicles Symposium 2000

Dearborn (MI), USA • October 3-5, 2000

\title{
Pavement Boundary Detection Via Circular Shape Models
}

\author{
Bing $\mathrm{Ma}^{*}$ \\ Department of EECS \\ University of Michigan \\ Ann Arbor, MI 48109 \\ bingm@umich.edu
}

\author{
Sridhar Lakshmanan \\ Department of ECE \\ University of Michigan - Dearborn \\ Dearborn, MI 48128 \\ lakshman@umich.edu
}

\author{
Alfred O. Hero \\ Department of EECS \\ University of Michigan \\ Ann Arbor, MI 48109 \\ hero@eecs.umich.edu
}

\begin{abstract}
Automated detection of pavement boundaries is an important enabling technology in a number of intelligent vehicle applications. Many state of art systems for detecting and tracking pavement boundaries use a priori shape models to describe the appearance of these boundaries.

Several types of shape models have been employed, and the model choice is usually made from the standpoint of accommodating all possible variations (in width, orientation, curvature, tilt, etc.) of the pavement boundaries relative to the host vehicle. Polynomial (quadratic or cubic) shape models are the ones of choice. This paper describes a circular shape model for detecting pavement boundaries. Indeed the polynomial shape models are intended as an approximation to the circular model, but the circular model itself has never been used before.

This paper shows that the circular shape models enjoy several critical advantages over the polynomial models without any additional increase in model complexity (i.e., number of model parameters): the model parameters are all of the same units, even a small change to any one parameter results in a uniformly different shape appearance, and as a result the associated shape matching problem (the one of matching various pavement shapes to the observed image) is considerably better conditioned than the corresponding problem with polynomial shape models. Our application domain is one of road/pavement boundary estimation based on image data from a high-resolution multibeam $77 \mathrm{GHz}$ millimeter-wave radar. A successful solution to this problem will impact a number of driver assistance systems, such as road departure warning, forward collision warning, etc.
\end{abstract}

- Research has been sponsored by the National Automotive Center at U.S. Army TACOM, and the Defense Advanced Projects Agency.

Proceedings of the 2000 Intelligent Vehicles Conference, The Ritz-Carlton Hotel, Dearborn, MI, USA, October 3-5, 2000.

$0-7803-6363-9 / 00 / \$ 10.00 \odot 2000$ IEEE
Keywords circular shape model, condition number, pavement boundary detection, deformable templates, millimeter-wave radar imaging, multisensor fusion

\section{Introduction}

Automated detection of pavement boundaries is an important enabling technology in a number of intelligent vehicle applications [1]. Two examples of its critical role are provided below. In the application of drowsy driver warning, knowledge of the pavement boundaries relative to the vehicle enables a driver assistance system to determine if the driver is running off the road. In the application of forward collision warning, pavement boundaries help disambiguate potential collision threats in terms of their relevance to the vehicle's intended path [1].

Over the years, pavement boundary detection systems have enjoyed systematic improvements to their performance. To the point that now there are studies underway to compare the performance of several systems side-by-side, and commercial availability of several such systems is imminent. While this is indeed a positive development, and feather in the cap for the intelligent vehicles community, systematic assessment of the system's performance is sadly lacking. Compared to the volume of papers on various systems of pavement detection and tracking the number of papers that provide frameworks for assessing their performance is miniscule.

This paper is an out growth of performance assessment effort that was previously reported [2]. This previous study concerned the fusion of images obtained simultaneously by radar and vision sensors. Using information from both sensors, the problem of locating lane and pavement boundaries was attempted. Central to the fusion methodology is an assessment of accuracy (i.e., confidence) of the lane and pavement boundary estimates. Alas, this was an elusive problem, because of the inherent incompatibility (in scale) between the image plane and ground plane parameters and the ill-conditioning of the image plane and the 
ground plane matching functions. We chose to attack the second problem first, as we found that to be particularly troubling given the wide acceptance of the image plane and ground plane matching functions used in $[3,4]$ within the intelligent vehicles and computer vision community. We refer the reader to [2] for more details.

It is generally accepted that the performance of state of art pavement boundary detection and tracking systems such as [1-8] - is directly attributed to the use of $a$ priori shape models to describe the appearance of these boundaries. The use of prior shape models allows these systems to reject false boundaries (such as entry/exit ramps) and also overcome image clutter (shadows) and occlusion. Polynomial (quadratic or cubic) shape models are the ones of choice, as they are known to parsimoniously accommodate all possible shape variations (width, orientation, curvature, tilt, etc.) of the pavement boundaries relative to the host vehicle [2-8]. These polynomials are meant to be approximations to a more accurate circular model, although the circular model itself has never been used.

This paper shows that the original circular shape model has several critical advantages over the polynomial models, without any additional increase in model complexity (i.e., number of model parameters):

- Model parameters are of same units - The circular model parameters are all in units of length, unlike parabolic approximations where the curvature parameter is in units of inverse length, the orientation parameter is dimensionless, and the offset parameter is in units of length.

- A small change in any parameter results uniform shape change - Even a small change to any one circular model parameter results in the corresponding pavement boundary shape changing over the entire image. Contrast that to the parabolic approximation, where even a substantial change in the curvature parameter results in the pavement boundary shape changing only at distances far away from the host vehicle.

- The shape matching problem is better conditioned - As a direct result of the above two properties, we report that the same matching function, in our case the log-normal function, appears to be better conditioned with respect to the circular model parameters than the quadratic case. By better conditioned we mean its sensitivity to the model parameters is more uniform in the case of the circular model than it is in the quadratic case.

Our application domain is one of road/pavement boundary estimation based on image data from a high-resolution multi-beam $77 \mathrm{GHz}$ millimeter-wave radar. We report that results obtained by using the circular model are just as accurate and robust as those obtained by using the quadratic model - see [4] for details.

Section 2 describes the circular shape model in detail. Section 3 provides a brief description of log-normal matching function. Section 4 reformulates the pavement detection problem in a Bayesian setting, followed by experimental results obtained using this new model in Section 5. The paper concludes with a view towards future work in Section 6.

\section{The circular shape model}

As mentioned earlier, prior work on pavement detection has focused on the use of polynomial shape models. For example, in references [3] and [4] pavement boundaries are described as parabolic curves on a flat earth,

$$
x=\frac{1}{2} k y^{2}+m y+b_{L, R}
$$

where the parameter $k$ is the road curvature, $m$ the tangential orientation of the road, and $b_{L, R}$ the offsets of left and right pavement boundaries, respectively, with respect to the host vehicle. While in reality the radius of curvature and tangential orientation of left and right pavement boundaries will differ slightly, constraining the left and right pavement boundaries to have the same parameters $k$ and $m$ closely approximates the actual pavement boundary shapes for all but very small radii of curvature roads. So, it is assumed that concentric pavement boundaries share the same parameters $k$ and $m$.

We mention this previously used model as a canonical example of what is wrong! Upon describing the circular model, we will attempt a systematic comparison of the two models.

It is common knowledge that in the United States public highway system pavement and lane boundaries are laid as concentric circles, at least over small segments. In this paper we propose to directly use a circular model instead of a parabolic approximation to it. In our application domain, a typical road scenario can be modeled by an intersection of a cone with two concentric circles. The cone represents the field-of-view of the radar sensor, and the two circles represent the left and right pavement boundaries. Assuming that the apex of the cone is at the origin $(0,0)$, we represent the coordinates $(x, y)$ of the pavement boundaries by circles centered at $\left(x_{c}, y_{c}\right)$ with radii $r_{L}$ and $r_{R}$, respectively

$$
\left(x-x_{c}\right)^{2}+\left(y-y_{c}\right)^{2}=r_{L, R}^{2}
$$

See Figure 1 for an illustration of the circular model.

There are several advantages of the new parameterization over the old one. The new model better reflects the road 


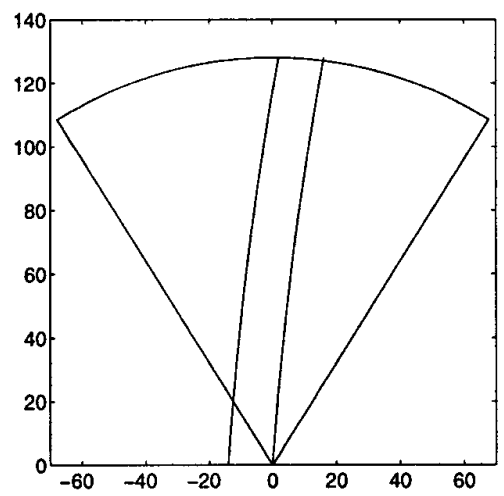

(a) Field-of-view of the radar sensor

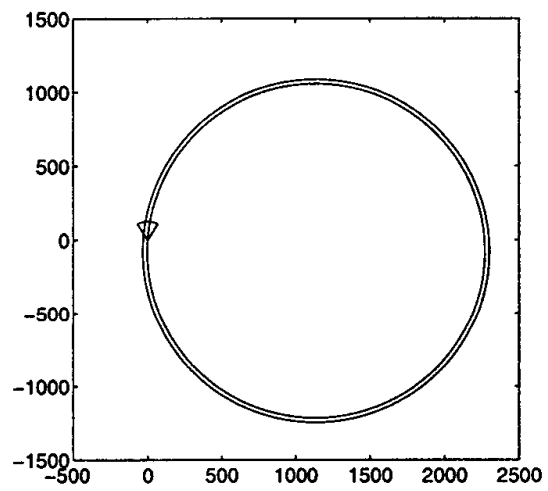

(b) Complete plot of the circular shape model

Figure 1: A typical road scenario in a radar image

shape of the real world. The four parameters $\left\{x_{c}, y_{c}, r_{L}, r_{R}\right\}$ have the same units - that of length. In addition, the ranges of feasible values for $x_{c}, y_{c}, r_{L}, r_{R}$ have the same order of magnitude. Let $\underline{\theta}_{c}=\left\{x_{c}, y_{c}, r_{L}, r_{R}\right\}$ and $\underline{\theta}_{q}=\left\{k, m, b_{L}, b_{R}\right\}$ denote the parameters of the circular and quadratic models, respectively. Note that their number of parameters are the same, or in other words neither model is more complex to describe than the other.

What sets the apart are the constraints that $\underline{\theta}_{c}$ and $\underline{\theta}_{q}$ have to satisfy in order to result in a "feasible" pair of left and right pavement boundaries. In the quadratic case, the feasibility region is a hypercube with respect to the model parameters $\left\{k, m, b_{L}, b_{R}\right\}$. In the circular model, the feasibility region is not so simple, although just as easily enforced:

1. The two circles must intersect the cone. That is, the cone cannot be totally inside the inner circle, neither can it be outside the outer circle (see Figure $2^{1}$ ). The constraint on the model parameters is

$$
r_{L}-p_{L} \leq x_{c}^{2}+y_{c}^{2} \leq r_{R}+p_{R}
$$

where $p_{L}$ and $p_{R}$ are two appropriately selected positive numbers that will allow the corresponding pavement boundaries to both be offset to either the left or the right of origin. Of course, the left boundary can be offset to the left of the origin, and the right boundary to its right.

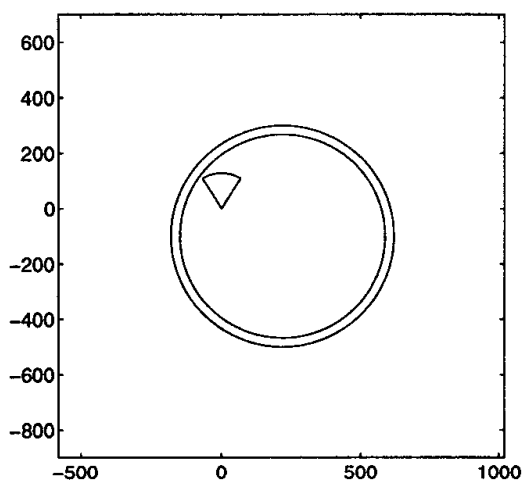

(a)

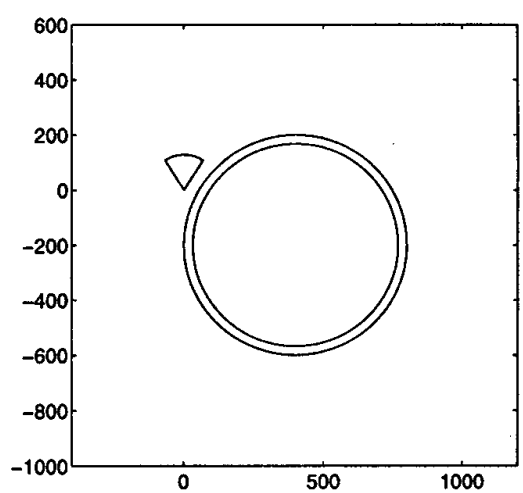

(b)

Figure 2: Cases where circles do not intersect the cone

2. The radar data is acquired under the assumption that the host vehicle is still within the road (or at least the shoulder). As such, the cases shown in Figure 3 are not realistic as they correspond to scenarios when the

\footnotetext{
${ }^{1}$ The plots in Figures 2 and 3 only demonstrate the relationship between the circles and the cone. In order to make the plots easily readable, the size of the cone is enlarged and the width of the road, while the size of the circles is decreased.
}

\section{6}




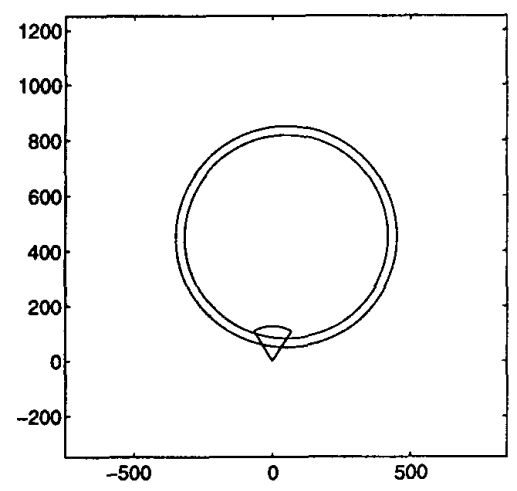

(a)

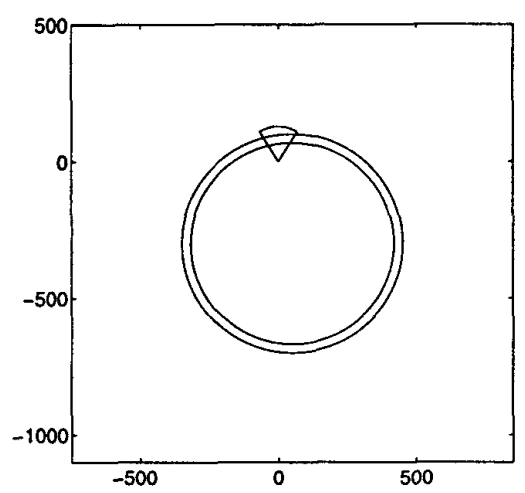

(b)

Figure 3: Cases where circles intersect the cone in an infeasible way

host vehicle is entirely off the road. The corresponding constraint on the model parameters is

$$
\alpha_{\min } \leq \operatorname{atan}\left(\frac{y_{c}}{x_{c}}\right) \leq \alpha_{\max }
$$

3. Finally, the road width has to be within a minimum and maximum limit,

$$
W_{\min } \leq r_{R}-r_{L} \leq W_{\max }
$$

The shape model (2) and the constraints (3), (4) and (5) constitute the prior knowledge regarding the pavement boundaries, resulting in a so-called prior pdf:

$$
\begin{aligned}
P\left(\underline{\theta}_{c}\right)= & \frac{1}{\gamma} \times I_{\left[r_{L}-p_{L}, r_{R}+p_{R}\right]}\left(x_{c}^{2}+y_{c}^{2}\right) \\
& \times I_{\left[\alpha_{\min }, \alpha_{\max }\right]}\left(\operatorname{atan}\left(\frac{y_{c}}{x_{c}}\right)\right) \\
& \times I_{\left[W_{\min }, W_{\max }\right]}\left(r_{R}-r_{L}\right)
\end{aligned}
$$

where $\gamma$ is a normalizing constant and $I_{A}(x)$ is an indicator function,

$$
I_{A}(x)= \begin{cases}1, & \text { if } x \in A \\ 0, & \text { otherwise }\end{cases}
$$

This prior pdf is empirically derived, and it is simply a uniform distribution over the space of feasible model parameters, and zero elsewhere.

\section{Imaging Likelihoods}

The millimeter-wave radar imaging process is very complicated - see [9] for details. In a radar image of a typical road, the pavement boundaries separate the image into three relatively homogeneous regions - the pavement surface, the left side of the pavement, and the right side of the pavement. It is accepted that the distribution of radar returns within each of these homogeneous regions is heavily tailed, and as such well modeled by an i.i.d. log-normal pdf. In other words, the probability of observing a particular radar image $Z=z$, given a hypothetical set of pavement boundary locations described by $\underline{\theta}_{c}$ is:

$$
\begin{aligned}
P\left(z \mid \underline{\theta}_{c}\right)= & \prod_{(x, y)} \frac{1}{z_{x y} \sqrt{2 \pi \sigma_{x y}^{2}\left(\underline{\theta}_{c}\right)}} \\
& \exp \left\{-\frac{1}{2 \sigma_{x y}^{2}\left(\underline{\theta}_{c}\right)}\left(\log z_{x y}-\mu_{x y}\left(\underline{\theta}_{c}\right)\right)^{2}\right\}
\end{aligned}
$$

where $\mu_{x y}, \sigma_{x y}^{2}$ denote the mean and variance of the radar return from the region where the pixel $(x, y)$ is located. See [4] for details.

\section{Bayesian estimation and boundary finding}

Using the prior and the likelihood pdfs in a Bayesian setting, the pavement boundary detection problem can be reformulated as a maximum a posteriori (MAP) estimation problem,

$$
\begin{aligned}
\underline{\hat{\theta}}_{c} & =\arg \max _{\underline{\theta}} P\left(\underline{\theta}_{c} \mid Z=z\right) \\
& =\arg \max _{\underline{\theta}} P\left(Z=z \mid \underline{\theta}_{c}\right) P\left(\underline{\theta}_{c}\right)
\end{aligned}
$$

Finding the MAP estimate is a non-trivial problem in itself, since the function $P\left(\underline{\theta}_{c} \mid Z=z\right)$ is multi-modal. The only sure method for finding the MAP estimate is exhaustive search, but it comes at the cost of unacceptable computation resources. In our case, the problem is especially hard since none of the parameters have an upper bound. In other words, there is no easy method for uniformly sampling the search space. To overcome this problem, we adopt a multi-resolution space switching pseudo-exhaustive search method instead: 
1. Constrain the parameters $\underline{\theta}_{c}^{\prime}=\left\{\frac{1}{r_{L}}, \frac{1}{r_{R}}, x_{c}, y_{c}\right\}$ in appropriate ranges. Note that although $r_{L}$ and $r_{R}$ do not have an upper bound, their inverse do!

2. Assign coarse step sizes $\Delta \underline{\theta}^{\prime}$ for the parameters in $\underline{\theta}_{c}^{\prime}$ and find $\underline{\hat{\theta}}_{c}^{\prime}$ by exhaustively maximizing the objective function in (8) over this coarse search space.

3. Once this coarse maximum $\underline{\hat{\theta}}_{c}^{\prime}$ is found, find the corresponding original parameters $\underline{\theta}_{c}$.

4. Set $\mathrm{i}=1$ and $\underline{\theta}_{c}^{(i)}=\underline{\theta}_{c}$ from the previous step. Centered at $\underline{\theta}_{c}^{(i)}$ and within a small constrained search space resolved using step size $\Delta \underline{\theta}^{(i)}$, find the parameters $\hat{\hat{\theta}}_{c}^{(i+1)}$ by exhaustively maximizing (8) over this smaller and finer space.

5. Set $\mathrm{i}=\mathrm{i}+1$. Choose $\Delta \underline{\theta}^{(i)}=\Delta \underline{\theta}^{(i-1)} / 2$. Repeat the previous step until the desired parameter grid size has reached.

The final estimated result is taken as the global MAP estimate.

\section{Experimental results}

We have applied the above algorithm to locate pavement boundaries in actual ground-level millimeter-wave radar images. These images were all obtained from a sensor platform mounted on top of WOLVERINE I (a selfcontained test-bed HMMWV). The platform contains a $77 \mathrm{GHz}$ FMCW radar sensor which has range and azimuthal resolution of $0.5 \mathrm{~m}$ and $1^{\circ}$, respectively. The radar's range extends out to $128 \mathrm{~m}$ and spans a $64^{\circ}$ horizontal field-of-view. Shown in Figures 4(a) and (b) are a pair of original radar image and accompanying visual image. ${ }^{2}$ The pavement boundary detection results are shown in Figure 4(c), where the white squares show the boundary detected with the proposed circular shape model and the white crosses show the boundary detected with the quadratic shape model addressed in [4]. Note that in both cases the likelihood function is the same (7). Figure 5 shows the detection results obtained for another image pair. The detection results using the new model are comparable in accuracy to the old model.

The principal advantage gained by employing the new model, as mentioned before, is in a better conditioned final estimate. To give a quantitative quantitative comparison, for each image in a database that contains 25 image pairs, at the estimated parameter points we calculate the ratio of the best and worst sensitivity of the objective function with

\footnotetext{
${ }^{2}$ The goal of also displaying visual images is just to give readers a feel for the ground truth. In this work, however, we only make use of the radar images.
}

respect to the model parameters. The average ratio is 56.67 for the circular shape model compared to $9.45 \times 10^{6}$ for the quadratic shape model.

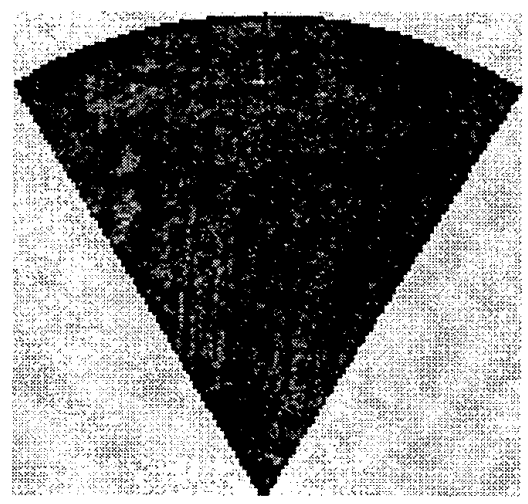

(a) Radar image

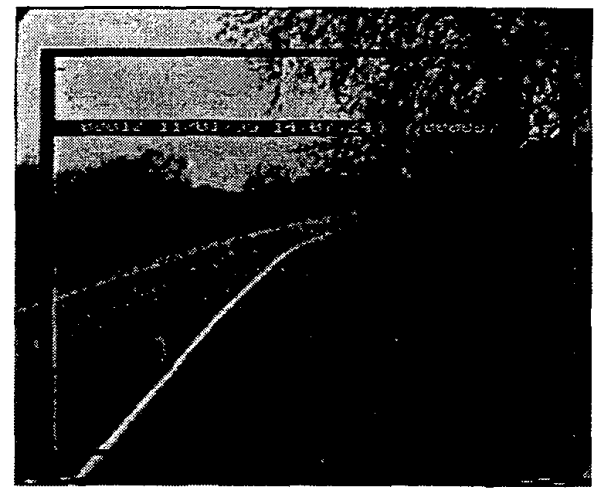

(b) Visual image

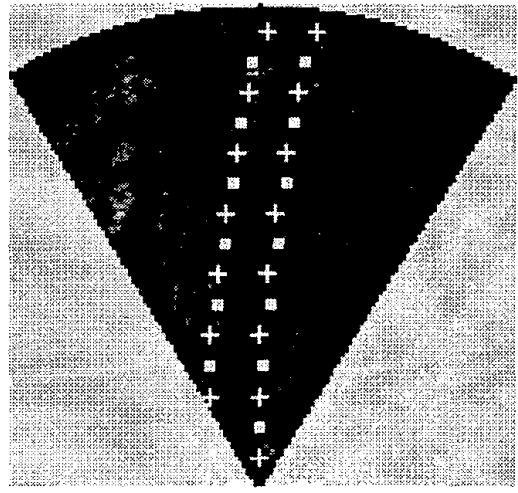

(c) Pavement boundary detection results

Figure 4: Pavement boundary detection using radar images 


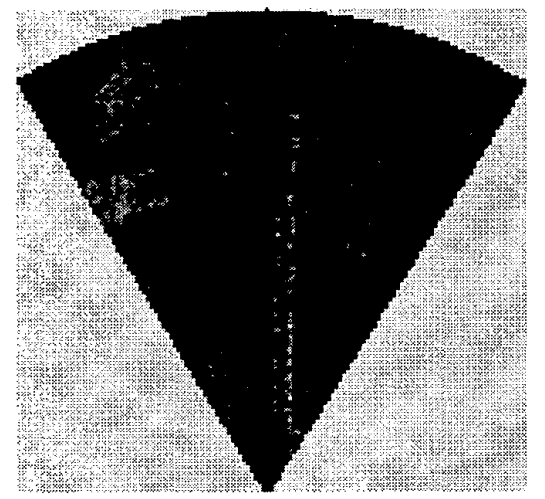

(a) Radar image

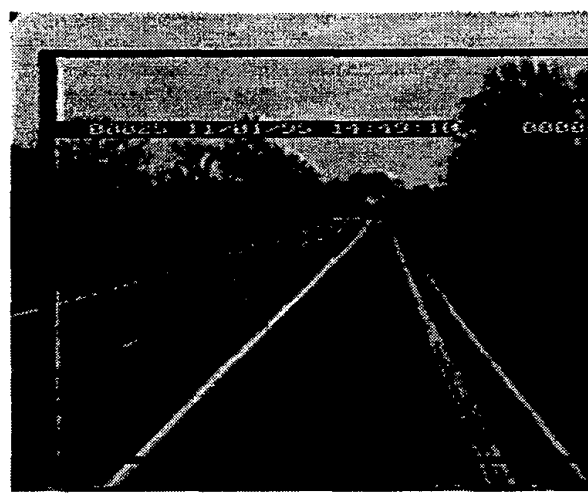

(b) Visual image

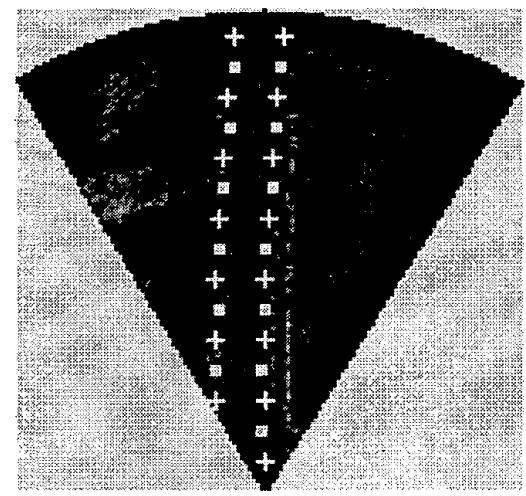

(c) Pavement boundary detection results

Figure 5: Pavement boundary detection using radar images

\section{Conclusions and future work}

We proposed a hitherto unused shape model to parameterize pavement boundaries. The paper showed that the circular shape models enjoy several critical advantages over the polynomial models without any additional increase in model complexity, while giving comparable estimation results.

We are especially encouraged by these results as we are poised to adapt this model for the problem of detecting lanes in images acquired from a vision sensor. We anticipate that the multi-sensor fusion problem we described earlier may not be as elusive any longer.

\section{References}

[1] Procedings of IEEE Intelligent Vehicles Symposium, 1998.

[2] B. Ma, S. Lakshmanan, and A. Hero, "Simultaneous detection of lane and pavement boundaries using model-based multisensor fusion," Submited to IEEE Trans. Intelligent Transportation Systems, Special Issue on Intelligent Vehicles.

[3] K. C. Kluge and S. Lakshmanan, "A deformabletemplate approach to lane detection," in Proc. Intell. Vehic. '95 Symp., Sept. 1995.

[4] B. Ma, S. Lakshmanan, and A. Hero, "Detection of curved road edges in radar images via deformable templates," in Proc. IEEE. Intl. Conf. Image Proc., 1997.

[5] E. D. Dickmanns and V. Graefe, "Applications of dynamic monocular machine vision," Machine Vision and Applications, pp. 241-261, 1988.

[6] E. D. Dickmanns and B. D. Mysliwetz, "Recursive 3-d road and relative ego-state recognition," IEEE Trans. Pattern Anal. Mach. Intell., vol. 14, no. 2, pp. 199-213, Feb. 1992.

[7] K. C. Kluge, "Extracting road curvature and orientation from image edge points without perceptual grouping into features," in Proc. Intell. Vehic. '94 Symp., Sept. 1994, pp. 109-114.

[8] K. Kluge and C. Thorpe, "The yarf system for visionbased road following," Mathematical and Computer Modelling, vol. 22, no. 4-7, pp. 213-234, Aug - Oct 1995.

[9] K. Kaliyaperumal, S. Lakshmanan, and K. Kluge, "Detecting roads and obstacles in radar images," to appear in IEEE Transactions on Vehicular Technology, 2000. 